A previous audit demonstrated that the guidance for target saturations was not being followed appropriately in all cases.

Methods Our aim was to review compliance with oxygen targeting prescriptions and the alarm limits used during saturation monitoring.

We collected data over three consecutive weeks during December 2011 for all babies on the neonatal unit who were monitored $(\mathrm{n}=102)$.

Results Compared to the previous audit we found there was an improvement in the set alarm limits to target oxygen within the guidelines to be correct $88 \%$ versus $69 \%$. However compliance with oxygen prescribing was suboptimal with $78 \%$ at best.

Conclusions Despite a change in the target saturation guidelines compliance with saturation alarm limits has improved. However further work is needed to ensure that all babies have their alarm limits set within the target range and also have their requirements for oxygen prescribed as per trust guidance.

It is also important to remember that setting the alarms correctly is only a step in oxygen targeting and that ideally the percentage of time in the target range should also be assessed.

\section{NOISE POLLUTION IN INTENSIVE CARE UNITS AND EMERGENCY WARDS}

doi:10.1136/archdischild-2012-302724.1629

G Khademi, M Roudi, AS Farhat. Mashhad University of Medical Sciences, Mashhad, Iran

Technology development has increased sound level beyond standards in hospitals. Such pollution undoubtedly has negative effects on staff heath and quality of care accordingly. Our goal with this study carried out to investigate noise levels in intensive care and emergency wards in Emam Reza hospital, at Mashhad University, Iran.

Materials and Methods This was a prospective study that performed on measuring of sound level of 10 intensive care and emergency wards of Imam Reza Hospital in a 30-minute-interval of AM working shift at one meter distance from nursing stations getting maximum sound level $\left(\mathrm{L}_{\max }\right)$ and Average Sound Level $\left(\mathrm{L}_{\text {eq }}\right)$.

Results Lmax in most wards were between $85-86 \mathrm{~dB}$. Leq in all wards were between $60.2 \mathrm{~dB}$. Average Leq in emergency wards was $62.2 \mathrm{~dB}$ without any justification. $\left(\mathrm{L}_{\mathrm{eq}}\right.$ ). in NICU was $60.8 \mathrm{~dB}$ (beyond standard level).In most of wards peak of noise were between 9:3011 AM. $\mathrm{L}_{\text {eq }}$ in all wards were more exceeded the standards levels.

Conclusion The average of sound levels in our wards remarkably exceeds the standards levels. The hospital staff should be aware of this noise level and its effects .

\section{CENTRAL VEIN CATHETER AND BLOODSTREAM INFECTION IN A PICU: CLABSI OR CRBSI?}

doi:10.1136/archdischild-2012-302724.1630

EA Volakli, S Stabouli, L Vogiatzi, A Tsolaki, K Skoumis, M Dimitriadou, M Sdougka. Pediatric Intensive Care Unit, Hippokratio General Hospital, Thessaloníki, Greece

Aims To differentiate between Central Line Associated Blood Stream Infections (CLABSI) and Catheter Related Blood Stream Infections (CRBSI) where the same microorganism is identified on catheter tip cultures and the bloodstream, in Pediatric Intensive Care Unit (PICU) patients.

Methods Prospective 1 year study of CLABSI (group 1- all patients with a CVC in situ) and CRBSI (group 2-only first, temporary CVC, inserted in PICU, during first admission). CLABSI and CRBSI rates were estimated according standard criteria. CVC insertion and maintenance was under local protocols (simple polyurethane, multiple lumen, plus chlorexidine-gluconate- impregnated sponge, stayed as long as needed if they were functioning without evidence of local or systemic complications).

Results 91 patients have had a total of 136 CVCs insertions. 112 temporary and 24 permanent catheters were evaluated for CLABSI whereas 73 CVCs were evaluated for CRBSI. Mean catheter days in group I was $11.95 \pm 12.48$ and 20 cases of CLABSI were recorded in 1626 catheter days, given a CLABSI rate of $12.3: 1000$, whereas mean catheter days in group 2 was 9.30 5.23 with 1 case of CRBSI in 679 catheter days, given a CRBSI rate of 1.47:1000.11 Gram -, 8 Gram + and 1 fungal infection were recorded in group 1 , and only $1 \mathrm{Gram}$ infection in group 2 .

Conclusions Strict adherence to protocols in group 2 led to much lower CRBSI compared to CLABSI. Gram - infections predominated. Reducing CLABSI and CRBSI over time, after implementation of bundles of care, should be the goal.

\section{CHANGE OF HABITS AFTER YEARS OF EVIDENCE: A QUESTIONNAIRE ON NEONATAL CARE}

doi:10.1136/archdischild-2012-302724.1631

${ }^{1} \mathrm{H}$ Küster, ${ }^{2} \mathrm{~S}$ Arenz, ${ }^{2} \mathrm{~T}$ Arenz, ${ }^{3} \mathrm{EM}$ Gerstl, ${ }^{2} \mathrm{H}$ Manser, ${ }^{2} \mathrm{M}$ Nelle, ${ }^{2} \mathrm{R}$ Gerull. ${ }^{2}$ Neonatology, University Children's Hospital, Göttingen, Germany; 2 Pediatrics, University Children's Hospital, Berne, Switzerland: ${ }^{3}$ Pediatrics, Kinderklinik Dritter Orden, Passau, Germany

Background and Aims Evidence based knowledge in neonatal care has substantially increased during the last years. We tried to evaluate how well guidelines and evidence from studies have been implemented into clinical practice during the last 15 years.

Methods Detailed questionnaires were sent 15 years apart to all neonatal units potentially treating VLBW infants in Germany (1995) and all German speaking countries (2010).

Results The response rate was 66\% both times. Whereas in 1995 $2 / 3$ of the units used $100 \%$ oxygen and $1 / 450 \%$ to start respiratory support in the delivery room, in 2010 only $3 \%$ and $5 \%$ of the units used $100 \%$ and $50 \%$ oxygen, respectively. Caffeine and Theophylline were used to treat apnoea of prematurity by $50 \%$ and $87 \%$ of the units in 1995 compared to $96 \%$ and $10 \%$ in 2010, respectively. Pasteurization of breast milk was done in 24\% of the units in 1995 compared to $53 \%$ in 2010 . In $1995,37 \%$ of the units routinely used erythropoietin in ELBW infants compared to $27 \%$ in 2010.

Conclusions Treatment of VLBW infants changed significantly within the last 15 years and some of the existing guidelines and evidence seem to be transferred into clinical practice in most units.

\section{QUALITY CLINICAL IMPROVEMENT: PROSPECTIVE TEST OF CHANGE}

doi:10.1136/archdischild-2012-302724.1632

'AA Onibere, '2 Ali, 3J Egyepong. 'Luton \& Dunstable University NHS Trust, Neonatal Unit, Luton; ${ }^{2}$ Department of Paediatrics, Bedford General Hospital, Bedford: ${ }^{3}$ Neonatal Intensive Care, Luton \& Dunstable University Hospital, Luton, UK

Background Change is a fundamental component of continuous quality improvement. Surfactant clearly works better the earlier it is given in at risk babies. Previously, surfactant was given after transfer to the NICU due to concerns such as the grade of doctors who were present during these deliveries and endotracheal (ETT) position. With the introduction of resident consultants, the NICU service became a fully consultant-delivered care and therefore always present at the stabilisation of these infants.

Audit of Practice Showed Surfactant administration at mean of $39 \mathrm{~min}$ ( 9 min outside our set protocol time), with wide variability.

Aims

- Feasibility and safety issues of giving surfactant right after intubation on Delivery suite,

Mean time to surfactant administration and ETT position postchange. 